\title{
Two-Dimension Echocardiographic Assessment of Left Atrial Volumetric Changes in Patients with Paroxysmal Atrial Fibrillation After Reversion to Sinus Rhythm Mohammed Osama Fathy Kayed, Yasser El-sayed Mohammed Hassan, * Reda Sayed Ahmed Albahnasy Abuhassan \\ Department of Cardiology, Faculty of Medicine, Al-Azhar University, Cairo, Egypt \\ * Corresponding author: Reda Sayed Ahmed Albahnasy Abuhassan, Mobile: (+20)01060097502, E-Mail: redaabahnasy@gmail.com
}

\section{ABSTRACT}

Background: Although often referred to as "the forgotten chamber", compared with left ventricle (LV), especially in the past years, the left atrium (LA) plays a critical role in the clinical expression and prognosis of patients with heart and cerebrovascular disease, as demonstrated by several studies.

Objective: The aim of the work was to assess the left atrium functional and volume changes during atrial fibrillation (AF) and after sinus rhythm cardioversion in patients with paroxysmal AF, to detect recovery of effective mechanical atrial function (EMAF) after cardioversion with preserved bioelectrical function.

Patients and methods: This prospective observational study included a total of 75 subjects divided on two groups: Group 1 (control) consisted of 25 apparently normal people with normal sinus rhythm, and Group 2 (patients) consisted of 50 patients with paroxysmal atrial fibrillation, attending at Damanhur Medical National Institute, for cardioversion. $25(50 \%)$ of patients were cardioverted pharmacologically either by amiodarone or propafenone and 25 (50\%) of patients were cardioverted by DC shock.

Results: Mean age of controls involved in the study was $44.60 \pm 9.82$ years. $56 \%$ of them were males. Mean age of patients was $51.40 \pm 7.23,54 \%$ of them were males. Left Anterior Descending Artery (LAD) showed a significant decreased after cardioversion and after one month. It showed insignificant decrease after 2 months. Number of patients who did not recovered effective mechanical atrial function (EMAF) after one month were 6 (12\%). Conclusion: Left atrial diameters and left atrial volumes increase in AF and showed significant reduction by the time after successfully cardioversion. Patients who were underwent DC shock had a slower recovery of atrial function and of LA volumes reduction, implying a greater degree of atrial dysfunction.

Keywords: 2D, EG, LV, LA, AF, HF.

\section{INTRODUCTION}

Although often referred to as "the forgotten chamber", compared with left ventricle (LV), especially in the past years, the left atrium (LA) plays a critical role in the clinical expression and prognosis of patients with heart and cerebrovascular disease, as demonstrated by several studies ${ }^{(\mathbf{1})}$.

Until recently, the LA had been subordinated to the LV, but cardiologists now recognize that LA function is indispensable to normal circulatory performance, conditioning the morbidity and mortality in several diseases. So, an early detection of LA dysfunction is anticipated to provide new insight into pathophysiology and clinical management of several conditions such as atrial fibrillation (AF), valvular heart disease, hypertension, heart failure (HF), and cardiomyopathy ${ }^{(1)}$.

Normal LA function can be divided into 3 distinct phases. During ventricular systole, the LA serves as a reservoir for blood drained by the pulmonary veins. During early ventricular diastole, the LA is a conduit for the pulmonary venous return. During late systole, the booster pump function of the LA completes the LV filling. Whereas the LA reservoir function is determined by atrial compliance, atrial relaxation, and contractility, as well as LV systolic function and end-systolic volume, the LA conduit function is influenced by LA compliance and LV relaxation and compliance, and the LA booster pump function is influenced by venous return, LV end-diastolic pressures, and systolic reserve ${ }^{(2)}$.

Because LA structural remodeling and $\mathrm{AF}$ are closely related, it is recommended that LA size and anatomy be assessed routinely in all AF patients ${ }^{(3)}$.

LA size is typically assessed with standard 2dimensional (2D) echocardiography. The LA anteroposterior diameter derived from a conventional parasternal long-axis view is used to estimate LA size. However, because asymmetrical remodeling occurs in LA dilation, it is recommended that LA volumes be assessed using a volumetric method ${ }^{(2)}$. The aim of the work was to assess the left atrium functional and volume changes during $\mathrm{AF}$ and after sinus rhythm cardioversion in patients with paroxysmal $\mathrm{AF}$, to detect recovery of effective mechanical atrial function (EMAF) after cardioversion with preserved bioelectrical function.

\section{PATIENTS AND METHODS}

This prospective observational study included a total of 75 subjects divided on two groups: Group 1 (control) consisted of 25 apparently normal people with normal sinus rhythm, and Group 2 (patients) consisted of 50 patients with paroxysmal atrial fibrillation, attending at Damanhur Medical National Institute. Approval of the ethical committee and a written informed consent from all the subjects were obtained. 


\section{Inclusion criteria:}

Patients with paroxysmal AF (selfterminating, in most cases within 48 hours). Some AF paroxysm may continue for up to 7 days.

Exclusion criteria: Patients with other types of AF including:

- First diagnosed AF

- Persistent AF

- Long standing persistent AF

- Permanent AF

\section{All patients were subjected to:}

I. Full history taking that included: age, gender, diabetes mellitus, hypertension, cigarette smoking, dyslipidemia and coronary artery disease.

II. Clinical examination that included: the estimation of heart rate, rhythm, pulse volume, respiratory rate, blood pressure, temperature, pulmonary rales, additional heart sounds, murmurs and pericardial rub.

\section{Investigations:}

\section{a) 12-lead electrocardiograms:}

12-lead electrocardiogram was recorded before drug administration. The diagnosis of AF was established based on 12-lead electrocardiogram (ECG) criteria Figure (1):

1. Absence of $\mathrm{p}$ waves.

2. Presence of irregular atrial electrical activity.

3. Irregular RR intervals

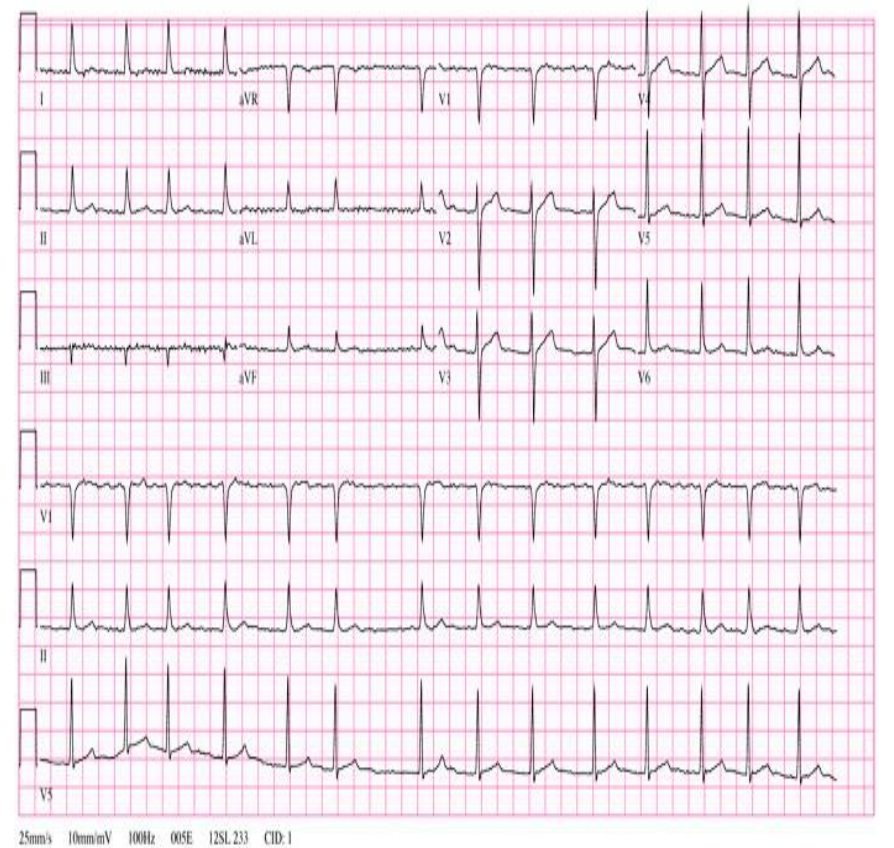

Figure (1): ECG diagnostic criteria of AF.

\section{B) Transthoracic echocardiogram:}

Transthoracic two-dimensional imaging and pulsed wave Doppler echocardiographic studies were obtained in all patients using.

Patients were examined in the supine and left lateral positions to obtain adequate images in the parasternal long and short axis views, the four, five and two chamber apical views, the apical long axis as well as in the subcostal views. Transthoracic echocardiography were performed before cardioversion, immediately after cardioversion, and after one month of cardioversion.

\section{The following items were assessed in each time:}

a) Left atrial diameters

b) Left atrial volumes

c) Effective mechanical atrial function (EMAF).

d) Echocardiographic follow-up after another one month if the EMAF not recovered. According to Manning et al. (5) EMAF defined Effective mechanic atrial function was defined as "the presence of AEF more than 7 dynes and of atrial filling wave peak velocity $\geq 0.5 \mathrm{~m} / \mathrm{s}$

\section{Cardioversion:}

The decision to perform electrical or pharmacologic cardioversion was made on the basis of the clinical situation, in consultation. For pharmacologic cardioversion, patients were hospitalized and monitored; Continuous electrocardiographic (ECG) monitoring was performed for several hours after the procedure to assess the maintenance of SR.

\section{Statistical analysis}

Recorded data were analyzed using the statistical package for social sciences, version 20.0 (SPSS Inc., Chicago, Illinois, USA). Quantitative data were expressed as mean \pm standard deviation (SD). Qualitative data were expressed as frequency and percentage.

\section{The following tests were done:}

- Independent-samples t-test of significance was used when comparing between two means.

- Chi-square $\left(\mathrm{x}^{2}\right)$ test of significance was used in order to compare proportions between two qualitative parameters.

- The confidence interval was set to $95 \%$ and the margin of error accepted was set to $5 \%$. The pvalue was considered significant as the following:

- Probability (P-value)

- P-value $<0.05$ was considered significant.

- P-value <0.001 was considered as highly significant.

- P-value >0.05 was considered insignificant.

\section{RESULTS}

The study included two groups; control group and patients group of paroxysmal AF. Mean age of controls was $44.60 \pm 9.82$ years. $56 \%$ of them were males. Mean age of patients was $51.40 \pm 7.23,54 \%$ of them were males. 
Table (1): Comparison between the two studied groups according to demographic data

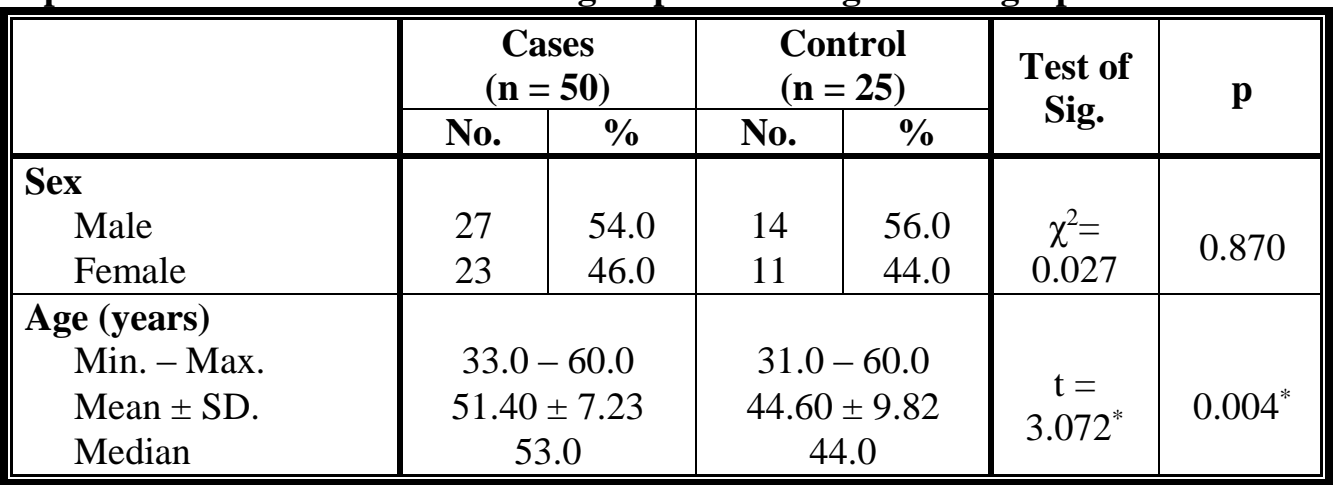

\section{$\chi^{2}$ : Chi square test}

t: Student t-test

$\mathrm{p}: \mathrm{p}$ value for comparing between the studied groups

*: Statistically significant at $\mathrm{p} \leq 0.05$

ECG of control group was regular rhythm with mean heart rate of $79.56 \pm 13.08$ with no any other significant changes. ECG of patients group showed irregular rhythm and absent P wave in $100 \%$ of patients, mean heart rate of $129.0 \pm 22.96$. Table (2)

Table (2): Comparison between the two studied groups according to ECG

\begin{tabular}{|c|c|c|c|c|c|c|}
\hline \multirow[t]{2}{*}{ ECG } & \multicolumn{2}{|c|}{$\begin{array}{c}\text { Cases } \\
(\mathbf{n}=\mathbf{5 0})\end{array}$} & \multicolumn{2}{|c|}{$\begin{array}{l}\text { Control } \\
(\mathbf{n}=25)\end{array}$} & \multirow{2}{*}{$\begin{array}{l}\text { Test of } \\
\text { Sig. }\end{array}$} & \multirow[t]{2}{*}{$\mathbf{P}$} \\
\hline & No. & $\%$ & No. & $\%$ & & \\
\hline $\begin{array}{l}\text { Rhythm } \\
\text { Regular } \\
\text { Irregular }\end{array}$ & $\begin{array}{c}0 \\
50\end{array}$ & $\begin{array}{c}0.0 \\
100.0\end{array}$ & $\begin{array}{c}25 \\
0\end{array}$ & $\begin{array}{c}100.0 \\
0.0\end{array}$ & $\begin{array}{c}\chi^{2}= \\
75.0^{*}\end{array}$ & $<0.001^{*}$ \\
\hline $\begin{array}{l}\text { HR } \\
\text { Min. - Max. } \\
\text { Mean } \pm \text { SD. } \\
\text { Median }\end{array}$ & \multicolumn{2}{|c|}{$\begin{array}{c}87.0-172.0 \\
129.0 \pm 22.96 \\
129.0\end{array}$} & \multicolumn{2}{|c|}{$\begin{array}{c}60.0-110.0 \\
79.56 \pm 13.08 \\
80.0\end{array}$} & $\begin{array}{c}\mathrm{t}= \\
11.867^{*}\end{array}$ & $<0.001^{*}$ \\
\hline Q waves & 5 & 10.0 & 0 & 0.0 & $\chi^{2}=2.679$ & ${ }^{F E} \mathrm{p}=0.161$ \\
\hline ST elevation & 0 & 0.0 & 0 & 0.0 & - & - \\
\hline P WAVE & 0 & 0.0 & 25 & 100.0 & $\chi^{2}=75.0^{*}$ & $<0.001^{*}$ \\
\hline $\begin{array}{l}\text { ST depression } \\
\text { No } \\
\text { V1 } \\
\text { V1,V2 } \\
\text { Lateral leads I,AVL } \\
\text { Anterior leads } \\
\text { Anterior and Lateral } \\
\text { Inferior }\end{array}$ & $\begin{array}{l}0 \\
0 \\
0 \\
0 \\
0 \\
0 \\
0\end{array}$ & $\begin{array}{l}0.0 \\
0.0 \\
0.0 \\
0.0 \\
0.0 \\
0.0 \\
0.0\end{array}$ & $\begin{array}{c}21 \\
1 \\
2 \\
1 \\
0 \\
0 \\
0\end{array}$ & $\begin{array}{l}0.0 \\
0.0 \\
0.0 \\
0.0 \\
0.0 \\
0.0 \\
0.0\end{array}$ & $\begin{array}{l}\chi^{2}=1.119 \\
\chi^{2}=2.027 \\
\chi^{2}=2.041 \\
\chi^{2}=2.027 \\
\chi^{2}=0.507 \\
\chi^{2}=1.027 \\
\chi^{2}=0.507\end{array}$ & $\begin{array}{r}\mathrm{FE}_{\mathrm{p}}=0.429 \\
\mathrm{FE}_{\mathrm{p}}=0.333 \\
\mathrm{FE}_{\mathrm{p}}=0.495 \\
\mathrm{FE}_{\mathrm{p}}=0.333 \\
\mathrm{FE}_{\mathrm{p}}=1.000 \\
\mathrm{FE}_{\mathrm{p}}=0.550 \\
\mathrm{FE}_{\mathrm{p}} 1.000\end{array}$ \\
\hline $\begin{array}{l}\text { T wave Inversion } \\
\text { No } \\
\text { V1 } \\
\text { AVL } \\
\text { V1,V2 } \\
\text { V2,V3 } \\
\text { Lateral }\end{array}$ & $\begin{array}{c}42 \\
4 \\
2 \\
0 \\
1 \\
1\end{array}$ & $\begin{array}{l}84.0 \\
8.0 \\
4.0 \\
0.0 \\
2.0 \\
2.0\end{array}$ & $\begin{array}{c}22 \\
0 \\
0 \\
2 \\
0 \\
1\end{array}$ & $\begin{array}{c}88.0 \\
0.0 \\
0.0 \\
8.0 \\
0.0 \\
4.0\end{array}$ & $\begin{array}{l}\chi^{2}=0.213 \\
\chi^{2}=2.113 \\
\chi^{2}=1.027 \\
\chi^{2}=2.041 \\
\chi^{2}=0.507 \\
\chi^{2}=0.257\end{array}$ & $\begin{array}{r}{ }^{\mathrm{FE}} \mathrm{p}=0.644 \\
\mathrm{FE}_{\mathrm{p}}=0.294 \\
\mathrm{FE}_{\mathrm{p}}=0.550 \\
\mathrm{FE}_{\mathrm{p}}=0.495 \\
{ }_{\mathrm{FE}} \mathrm{p}=1.000 \\
{ }^{\mathrm{FE}_{\mathrm{p}}}=1.000\end{array}$ \\
\hline
\end{tabular}

$\chi^{2}$ : Chi square test

FE: Fisher Exact

t: Student t-test

$\mathrm{p}$ : $\mathrm{p}$ value for comparing between the studied groups $*$ : Statistically significant at $\mathrm{p} \leq 0.05$

\section{Volume minimum (Vmin):}

Follow up after cardioversonVmin showed significant reduction $(\mathrm{p} 1<0.001)$. After one month the Vmin showed significant reduction $(\mathrm{p} 1<0.001)$ and number of cases which did not recover EMF were $6(12 \%)$ which also showed significant reduction after 2 months follow up $(\mathrm{p} 1<0.001)$. Table (3) 
Table (3): Comparison between the two studied groups according to $\mathrm{V}$ min

\begin{tabular}{|c|c|c|c|c|c|}
\hline \multirow{3}{*}{$\operatorname{Vmin}(\mathbf{m l})$} & \multicolumn{4}{|c|}{ Cases } & \multirow{3}{*}{$\begin{array}{l}\text { Control } \\
(n=25)\end{array}$} \\
\hline & \multirow{2}{*}{$\begin{array}{c}1^{\text {st }} \\
(n=50)\end{array}$} & \multicolumn{3}{|c|}{ Cardioverson } & \\
\hline & & $\begin{array}{c}\text { Post } \\
(\mathbf{n}=\mathbf{5 0})\end{array}$ & $\begin{array}{l}1 \text { month } \\
(\mathbf{n}=50)\end{array}$ & $\begin{array}{c}2 \text { months } \\
(n=6)\end{array}$ & \\
\hline Min. - Max. & $24.90-30.20$ & $16.0-27.6$ & $10.90-25.10$ & $12.07-24.70$ & $11.30-25.20$ \\
\hline Mean \pm SD. & $28.19 \pm 1.72$ & $23.23 \pm 5.78$ & $18.82 \pm 4.89$ & $18.84 \pm 4.52$ & $18.98 \pm 4.84$ \\
\hline Median & 28.30 & 21.80 & 19.30 & 19.05 & 19.30 \\
\hline $\mathbf{t}(\mathbf{p})$ & $9.230^{*}\left(<0.001^{*}\right)$ & $3.162^{*}\left(0.002^{*}\right)$ & $0.137(0.892)$ & & \\
\hline Sig. bet. periods. & \multicolumn{3}{|c|}{$\mathrm{p}_{1}<0.001^{*}, \mathrm{p}_{2}<0.001^{*}, \mathrm{p}_{3}<0.001^{*}$} & & \\
\hline
\end{tabular}

\section{t: Student t-test}

$\mathrm{p}_{1}$ : $\mathrm{p}$ value for comparing between $\mathbf{1}^{\text {st }}$ and post

$\mathrm{p}_{2}$ : $\mathrm{p}$ value for comparing between $\mathbf{1}^{\text {st }}$ and $\mathbf{1}$ month

$\mathrm{p}_{3}: \mathrm{p}$ value for comparing between post and 1 month

Total atrial stroke volume (TA-SV):

Follow up after cardioversion TA-SV showed significant reduction $(\mathrm{p} 1<0.001)$. After one month the TA-SV showed significant reduction ( $1<0.001)$ and number of cases which did not recover EMF were $6(12 \%)$ which also showed significant reduction after 2 months follow up (p1<0.001), Table (4).

Table (4): Comparison between the two studied groups according to TA-SV

\begin{tabular}{|c|c|c|c|c|c|}
\hline \multirow{3}{*}{ TA-SV (mm) } & \multicolumn{4}{|c|}{ Cases } & \multirow{3}{*}{$\begin{array}{l}\text { Control } \\
(n=25)\end{array}$} \\
\hline & \multirow{2}{*}{$\begin{array}{c}1^{\text {st }} \\
(n=50)\end{array}$} & \multicolumn{3}{|c|}{ Cardioverson } & \\
\hline & & $\begin{array}{c}\text { Post } \\
(n=50)\end{array}$ & $\begin{array}{c}1 \text { month } \\
(n=50)\end{array}$ & $\begin{array}{c}2 \text { months } \\
(\mathrm{n}=6)\end{array}$ & \\
\hline Min. - Max. & $23.60-30.20$ & $15.0-29.80$ & $14.70-24.80$ & $15.40-23.90$ & $15.10-24.70$ \\
\hline Mean \pm SD. & $27.61 \pm 1.69$ & $24.02 \pm 4.45$ & $20.48 \pm 3.36$ & $19.88 \pm 3.35$ & $20.45 \pm 3.27$ \\
\hline Median & 27.70 & 24.60 & 20.80 & 20.31 & 19.90 \\
\hline $\mathbf{t}(\mathbf{p})$ & $10.277^{*}\left(<0.001^{*}\right)$ & $3.554^{*}\left(0.001^{*}\right)$ & $0.030(0.976)$ & & \\
\hline Sig. bet. periods. & \multicolumn{3}{|c|}{$\mathrm{p}_{1}<0.001^{*}, \mathrm{p}_{2}<0.001^{*}, \mathrm{p}_{3}<0.001^{*}$} & & \\
\hline
\end{tabular}

t: Student t-test

$\mathrm{p}_{1}$ : $\mathrm{p}$ value for comparing between $\mathbf{1}^{\text {st }}$ and post

$\mathrm{p}_{2}$ : $\mathrm{p}$ value for comparing between $\mathbf{1}^{\text {st }}$ and $\mathbf{1}$ month

$\mathrm{p}_{3}$ : $\mathrm{p}$ value for comparing between post and 1 month

Total atrial emptying fraction (TA-EF) \%:

Follow up after cardioverson TA-EF \% mean was $54.89 \pm 6.33$ (insignificant reduction $\mathrm{p}=.088$ ) .after one month the TA-EF \% showed significant reduction $(\mathrm{p}=0.043)$ and number of cases which did not recover EMF were $6(12 \%)$ which also showed reduction after 2 months follow up. Table (5)

Table (5): Comparison between the two studied groups according toTA-EF

\begin{tabular}{|c|c|c|c|c|c|}
\hline \multirow{3}{*}{ TA-EF(\%) } & \multicolumn{4}{|c|}{ Cases } & \multirow{3}{*}{$\begin{array}{l}\text { Control } \\
(n=25)\end{array}$} \\
\hline & \multirow{2}{*}{$\begin{array}{c}1^{\text {st }} \\
(\mathbf{n}=\mathbf{5 0})\end{array}$} & \multicolumn{3}{|c|}{ Cardioverson } & \\
\hline & & $\begin{array}{c}\text { Post } \\
(n=50)\end{array}$ & $\begin{array}{c}1 \text { month } \\
(\mathrm{n}=50)\end{array}$ & $\begin{array}{c}2 \text { months } \\
(n=6)\end{array}$ & \\
\hline Min. - Max. & $41.60-69.80$ & $35.7-66.40$ & $40.90-67.30$ & $40.90-55.06$ & $40.90-65.80$ \\
\hline Mean \pm SD & $56.19 \pm 4.64$ & $54.89 \pm 6.33$ & $53.21 \pm 6.39$ & $52.63 \pm 5.75$ & $52.41 \pm 6.37$ \\
\hline Median & 56.85 & 51.05 & 52.01 & 55.03 & 53.32 \\
\hline $\mathbf{t}(\mathbf{p})$ & $2.923^{*}\left(0.005^{*}\right)$ & $1.597(0.115)$ & $0.512(0.610)$ & & \\
\hline Sig. bet. periods. & \multicolumn{3}{|c|}{$\mathrm{p}_{1}=0.088, \mathrm{p}_{2}=0.043^{*}, \mathrm{p}_{3}=1.000$} & & \\
\hline
\end{tabular}

\section{t: Student t-test}

$\mathrm{p}$ : $\mathrm{p}$ value for comparing between Cases and Control in each periods

$\mathrm{p}_{1}$ : $\mathrm{p}$ value for comparing between $\mathbf{1}^{\text {st }}$ and post

$\mathrm{p}_{2}$ : $\mathrm{p}$ value for comparing between $\mathbf{1}^{\text {st }}$ and $\mathbf{1}$ month

$\mathrm{p}_{3}$ : $\mathrm{p}$ value for comparing between post and 1 month 
The following table shows significant reduced AEF between patients underwent to cardioverson with DC shock and those with pharmacological group among all periods (immediately after cardioverson, after one month, and after two months). Table (6)

Table (6): Comparison between the three studied groups according to AEF

\begin{tabular}{|c|c|c|c|c|c|}
\hline \multirow[b]{2}{*}{ AEF (dynes) } & \multicolumn{2}{|c|}{ Cases $(n=50)$} & \multirow{2}{*}{$\begin{array}{l}\text { Control } \\
\text { group }\end{array}$} & \multirow{2}{*}{$\begin{array}{l}\text { Test of } \\
\text { sig. }\end{array}$} & \multirow[b]{2}{*}{$\mathbf{p}$} \\
\hline & $\begin{array}{c}\text { Pharmacological } \\
\text { group }\end{array}$ & $\begin{array}{c}\text { DC shock } \\
\text { group }\end{array}$ & & & \\
\hline $\begin{array}{l}\text { Post } \\
\text { Min. - Max. } \\
\text { Mean } \pm \text { SD. } \\
\text { Median } \\
\end{array}$ & $\begin{array}{c}(\mathbf{n}=\mathbf{2 5}) \\
5.90-13.20 \\
9.81 \pm 1.95 \\
10.40 \\
\end{array}$ & $\begin{array}{c}(\mathbf{n}=\mathbf{2 5}) \\
4.70-8.20 \\
6.32 \pm 0.93 \\
6.30\end{array}$ & $\begin{array}{c}(\mathbf{n}=\mathbf{2 5}) \\
8.60-16.50 \\
12.06 \pm 1.87 \\
11.40\end{array}$ & $\mathrm{H}=49.170^{*}$ & $<0.001^{*}$ \\
\hline Sig.bet.Grps & \multicolumn{3}{|c|}{$\mathrm{p}_{1}<0.001^{*}, \mathrm{p}_{2}=0.008^{*}, \mathrm{p}_{3}<0.001^{*}$} & & \\
\hline $\begin{array}{l}1 \text { month } \\
\text { Min. - Max. } \\
\text { Mean } \pm \text { SD. } \\
\text { Median }\end{array}$ & $\begin{array}{c}(\mathbf{n}=\mathbf{2 5}) \\
7.20-13.30 \\
10.19 \pm 1.73 \\
10.90\end{array}$ & $\begin{array}{c}(\mathbf{n}=\mathbf{2 5}) \\
4.90-9.70 \\
7.43 \pm 1.25 \\
7.60\end{array}$ & $\begin{array}{c}(\mathbf{n}=\mathbf{2 5}) \\
8.60-16.50 \\
12.06 \pm 1.87 \\
11.40\end{array}$ & $\mathrm{H}=43.684^{*}$ & $<0.001^{*}$ \\
\hline Sig.bet.Grps & \multicolumn{3}{|c|}{$\mathrm{p}_{1}<0.001^{*}, \mathrm{p}_{2}=0.011^{*}, \mathrm{p}_{3}<0.001^{*}$} & & \\
\hline $\begin{array}{l}2 \text { months } \\
\text { Min. - Max. }\end{array}$ & $\left(\mathbf{n}=\mathbf{1}^{\#}\right)$ & $\begin{array}{c}(\mathbf{n}=\mathbf{5}) \\
7.50-8.20\end{array}$ & $\begin{array}{c}(\mathbf{n}=\mathbf{2 5}) \\
8.60-16.50\end{array}$ & & \\
\hline $\begin{array}{l}\text { Mean } \pm \text { SD. } \\
\text { Median }\end{array}$ & 10.20 & $\begin{array}{c}7.88 \pm 0.28 \\
7.80 \\
\end{array}$ & $\begin{array}{c}12.06 \pm 1.87 \\
11.40 \\
\end{array}$ & $\mathrm{U}=0.0^{*}$ & $<0.001^{*}$ \\
\hline
\end{tabular}

H: H for Kruskal Wallis test, Pairwise comparison bet. each 2 groups was done using Post Hoc Test (Dunn's for multiple comparisons test), U: Mann Whitney test

$\mathrm{p}: \mathrm{p}$ value for comparing between the three studied groups

*: Statistically significant at $\mathrm{p} \leq 0.05$

The following table shows significant reduced peak A wave between patients underwent to cardioversion with DC shock and those with pharmacological group among all periods (immediately after cardioversion, after one month, and after two months). Table (7)

Table (7): Comparison between the three studied groups according to peak A wave

\begin{tabular}{|c|c|c|c|c|c|}
\hline \multirow[b]{2}{*}{ Peak A wave(ms) } & \multicolumn{2}{|c|}{ Cases $(n=50)$} & \multirow{2}{*}{$\begin{array}{l}\text { Control } \\
\text { group }\end{array}$} & \multirow{2}{*}{$\begin{array}{l}\text { Test of } \\
\text { sig. }\end{array}$} & \multirow[b]{2}{*}{$\mathbf{p}$} \\
\hline & $\begin{array}{c}\text { Pharmacological } \\
\text { group }\end{array}$ & $\begin{array}{l}\text { DC shock } \\
\text { group }\end{array}$ & & & \\
\hline \begin{tabular}{|l} 
Post \\
Min. - Max. \\
Mean \pm SD. \\
Median \\
\end{tabular} & $\begin{array}{c}(\mathbf{n}=\mathbf{2 5}) \\
29.10-64.50 \\
51.46 \pm 9.62 \\
50.70 \\
\end{array}$ & $\begin{array}{c}(\mathbf{n}=\mathbf{2 5}) \\
25.50-54.80 \\
41.44 \pm 9.78 \\
43.80 \\
\end{array}$ & $\begin{array}{c}(\mathbf{n}=\mathbf{2 5}) \\
57.40-79.40 \\
68.36 \pm 6.61 \\
68.60 \\
\end{array}$ & $\mathrm{~F}=59.896^{*}$ & $<0.001^{*}$ \\
\hline Sig.bet.Grps & \multicolumn{3}{|c|}{$\mathrm{p}_{1}<0.001^{*}, \mathrm{p}_{2}<0.001^{*}, \mathrm{p}_{3}<0.001^{*}$} & & \\
\hline \begin{tabular}{|l} 
month \\
Min. - Max. \\
Mean \pm SD. \\
Median \\
\end{tabular} & $\begin{array}{c}(\mathbf{n}=\mathbf{2 5}) \\
50.20-80.20 \\
67.97 \pm 8.71 \\
66.90 \\
\end{array}$ & $\begin{array}{c}(\mathbf{n}=\mathbf{2 5}) \\
43.30-70.40 \\
55.47 \pm 6.84 \\
55.40\end{array}$ & $\begin{array}{c}(\mathbf{n}=\mathbf{2 5}) \\
57.40-79.40 \\
68.36 \pm 6.61 \\
68.60 \\
\end{array}$ & $\mathrm{~F}=24.221^{*}$ & $<0.001^{*}$ \\
\hline Sig.bet.Grps & \multicolumn{3}{|c|}{$\mathrm{p}_{1}<0.001^{*}, \mathrm{p}_{2}=0.981, \mathrm{p}_{3}<0.001^{*}$} & & \\
\hline $\begin{array}{l}2 \text { months } \\
\text { Min. - Max. } \\
\text { Mean } \pm \text { SD. } \\
\text { Median }\end{array}$ & $\left(n=1^{\#}\right)$ & $\begin{array}{c}(\mathbf{n}=\mathbf{5}) \\
52.20-61.20 \\
56.58 \pm 3.87 \\
57.60\end{array}$ & $\begin{array}{c}(\mathbf{n}=\mathbf{2 5}) \\
57.40-79.40 \\
68.36 \pm 6.61 \\
68.60\end{array}$ & $\mathrm{t}=3.822^{*}$ & $0.001^{*}$ \\
\hline
\end{tabular}

F: $F$ for ANOVA test $t$ : Student t-test

$\mathrm{p}$ : $\mathrm{p}$ value for comparing between the three studied groups

*: Statistically significant at $\mathrm{p} \leq 0.05$ 


\section{DISCUSSION}

This study included only paroxysmal $\mathrm{AF}$ which defined as AF that terminates spontaneously or with intervention within 7 days of onset ${ }^{(4)}$. Episodes may recur with variable frequency and excluded other types of $\mathrm{AF}^{(3)}$.

In our study we included $58 \%$ of patients with hypertension which showed increased left atrial volume compared with hypertensive patients without paroxysmal AF. This agree with study by Tenekecioglu et al. ${ }^{\left({ }^{(6)}\right.}$. He also found that LA booster pump function was increased in hypertensive patients when compared to normotensive subjects, it was impaired in hypertensive subjects with PAF as compared with hypertensive patients without PAF.

Effective mechanical atrial function defined by Mattioli et al. ${ }^{(7)}$ as the presence of atrial filling waves (peak A wave) $\geq 0.5 \mathrm{~m} / \mathrm{s}$ and atrial ejection force (AEF) $\geq 7$ dynes.

As mentioned above we can detect EMAF by peak A wave velocity and atrial ejection force (AEF). The current study showed difference between modality of cardioversion between both two groups:(1) for pharmacological group AEF $<7$ dynes after cardioversion in 5 patients 20\%.Follow up after one month, all group was recovered EMAF, Peak A velocity $<50 \mathrm{~cm} / \mathrm{s}$ after cardioversion in 9 patients $36 \%$.Follow up after one month, all group was recovered. (2) for DC shock group, AEF $<7$ dynes after cardioversion in 18 patients $72 \%$. Follow up after one month 6 patients (24\%) were $<7$ dynes, after 2 months all group was recovered EMAF .Peak A velocity $<50 \mathrm{~cm} / \mathrm{s}$ after cardioversion in 16 patients $64 \%$. Follow up after one month 6 patients $(24 \%)<50$ $\mathrm{cm} / \mathrm{s}$, after 2 months all group was recovered EMAF.

A study by Mattioli et al. ${ }^{(7)}$ concluded that, the size of the left atrium decreased after restoration of sinus in $50 \%$ of patients reverted with DC shock. The comparison between patients with a normal mechanical atrial function and patients with reduced atrial function showed that a higher atrial ejection force was associated with a more marked reduction in LA size after restoration of sinus rhythm. A relation between LA volumes and atrial ejection force was observed in the group of patients with depressed atrial mechanical function $(\mathrm{r}=-0.78 ; \mathrm{p}<0.001)$. Thus, a depressed atrial mechanical function after cardioversion for $\mathrm{AF}$ was associated with a persistence of LA dilation.

Accordingly, the ASE/ESC has recommended quantification of LA size by biplane volumetric twodimensional echocardiography using either the Simpson method or the area-length method which are comparable in accuracy and reproducibility. Biplane LAV by two-dimensional echocardiography provides a more accurate and reproducible estimation of LA size when compared with reference standards such as magnetic resonance imaging and threedimensional echocardiography ${ }^{(\mathbf{8})}$.

The size of the left atrium is usually increased during AF. Post cardioversion was $46.71 \pm 8.27$ (significant decreased $\mathrm{p}<0.001$ ). After 1 month mean LAD was $39.40 \pm 2.30$ (significant decreased $p$ $<0.028$ ).after 2 months number of patient who were not recovered EMAF was 6 (12\%), mean LAD was $39.58 \pm 1.82$ (insignificant decreased).

Enlargement of LA is known to be related to aging, hypertension, and LV diastolic dysfunction, and is an independent factor for occurrence of $\mathrm{AF}^{(\mathbf{9})}$. In the present study, LAVmax was found to be an independently associated factor for paroxysmal $\mathrm{AF}$ even after adjusting for other factors such as aging, sex, and presence of hypertension.

In addition to LAVmax, LAVpreA and LAVmin were found to be greater in PAF than those in controls. Fatema et al. ${ }^{(10)}$ reported that not only LAVmax but also LAVmin were independent predicting factors for $\mathrm{AF}$, and, under these conditions, LAVmin might be better than LAVmax in terms of predicting value for AF. However, as pointed out in a previous and in our study, reproducibility for measurement of LAVmin was found not enough to use for clinical practice.

To characterize the three phases of LA activity, PA-SV and PA-EF were defined as indices for LA conduit function, AA-SV, AA-EF, for LA pump function, and AEI for LA reservoir function.

LA pump function: Both active atrial stroke volume (AA-SV) and active atrial empty fraction (AA-EF) defined as indices for LA pump function and cannot be assessed during PAF attack as pump function of left atrium is absent. However, after cardioversion we can assess it. After one month the AA-SV and AA-EF showed significant reduction $(\mathrm{p}<0.001)$ i.e. left atrium showed marked pump function improvement by the time after cardioversion.

LA reservoir function Atrial expansion index (AEI), total atrial stroke volume, and total atrial empty fraction named as LA reservoir function. These volume showed significant reduction by the time after cardioversion.

LA conduit function PA-SV and PA-EF were defined as indices for LA conduit function follow up after cardioversion both PA-SV and PA-EF showed significant reduction $(\mathrm{p}<0.001)$ and $(\mathrm{P}=.035)$ respectively.

Clinical Implications: This Study suggests that anticoagulant therapy after conversion of AFib is mandatory especially in patients who have undergone DC shock as a result of the increased embolic risk. 


\section{CONCLUSION}

It could be concluded that left atrial diameters and left atrial volumes increase in AF and showed significant reduction by the time after successfully cardioversion

This study suggests a direct relationship between the delay in the restoration of mechanic atrial function and a persistently dilated LA. This phenomenon is more evident after electric cardioversion and might be related to atrial electric remodeling.

This study showed a correlation between recovery of atrial function and the size of the LA. The temporal course of return of atrial contraction velocities to normal values is associated with a reduction of LA volumes.

Patients who were underwent DC shock had a slower recovery of atrial function and of LA volumes reduction, implying a greater degree of atrial dysfunction.

\section{RECOMMENDATIONS}

- Further researches in patients with all types of atrial fibrillation are needed.

- Closely monitoring patients of paroxysmal AF to include the patients who are spontaneously cardioverted to sinus rhythm.

- Further studies are needed with larger number of patients and more accurate methods for assessment of left atrium other than 2DEchocardiography.

\section{REFERENCES}

1. Roberta A, Salvatore CP, Pio C et al. (2014): Left Atrium by Echocardiography in Clinical Practice: From Conventional Methods to New Echocardiographic Techniques. Scientific World Journal, 2014: 451042.
2. Victoria D, Luigi DB, Melissa L et al. (2017): Structure and Function of the Left Atrium and Left Atrial Appendage. J Am Coll Cardiol., 70(25):31573172.

3. Kirchhof $\mathrm{P}$, Ammentorp $\mathrm{B}$, Darius $\mathrm{H}$ et al. (2014)Management of atrial fibrillation in seven European countries after the publication of the 2010 ESC Guidelines on atrial fibrillation Europace. Europace, 16(1):6-14.

4. Kirchhof P, Stefano B, Dipak K et al. (2016): ESC Guidelines for the management of atrial fibrillation developed in collaboration with EACTS. https://moh-

it.pure.elsevier.com/en/publications/2016-escguidelines-for-the-management-of-atrial-fibrillationdev-2

5. Manning WJ, Leeman DE, Gotch PJ et al. (1989): Pulsed Doppler evaluation of atrial mechanical function after electrical cardioversion of atrial fibrillation. J Am Coll Cardiol., 13:617-23

6. Tenekecioglu E, Vatansever A, Ozlem A et al. (2014): Disturbed Left Atrial Function is Associated with Paroxysmal Atrial Fibrillation in Hypertension. Arq Bras Cardiol., 102(3): 253-262.

7. Mattioli AV, Castelli A, Andria A et al. (1998): Clinical and echocardiographic features influencing recovery of atrial function after cardioversion of atrial fibrillation. Amerian Journal of Cardiology, 82(11): 1368-1371.

8. Maddukuri PV, Vieira ML, DeCastro $\mathrm{S}$ et al. (2006): What is the best approach for the assessment of left atrial size?, J Am Soc Echocardiogr., 19 : 1026-32.

9. Casaclang-Verzosa G, Gersh BJ and Tsang TS (2008): Structural and functional remodeling of the left atrium: clinical and therapeutic implications for atrial fibrillation Am Coll Cardiol., 51: 1-11

10. Fatema K, Barnes ME, Bailey KR et al. (2009): Echocardiogr Minimum vs. maximum left atrial volume for prediction of first atrial fibrillation or flutter in an elderly cohort: a prospective study. Eur J Echocardiogr., 10(2):282-6. 